\title{
Review
}

\section{Arrhythmia Monitoring for Risk Stratification in Hypertrophic Cardiomyopathy}

\author{
Darson Du, MD, ${ }^{a}$ Christopher O.Y. Li, MD, ${ }^{a}$ Kevin Ong, MD, ${ }^{a}$ Ashkan Parsa, MD, ${ }^{a}$ \\ Adaya Weissler-Snir, MD, ${ }^{b}$ Jeffrey B. Geske, MD, ${ }^{c}$ and Zachary Laksman, MSc, MD, FRCPC ${ }^{a}$ \\ ${ }^{a}$ Division of Cardiology, Department of Medicine, Faculty of Medicine, University of British Columbia, Vancouver, British Columbia, Canada \\ ${ }^{b}$ Division of Cardiology, Hartford Hospital, University of Connecticut, Hartford, Connecticut, USA \\ 'Department of Cardiovascular Medicine, Mayo Clinic, Rochester, Minnesota, USA
}

\begin{abstract}
Hypertrophic cardiomyopathy $(\mathrm{HCM})$ is the most common inherited cardiomyopathy, presenting significant clinical heterogeneity. Arrhythmia risk stratification and detection are critical components in the evaluation and management of all cases of HCM. The 2020 American Heart Association/American College of Cardiology HCM guidelines provide new recommendations for periodic 24-48-hour ambulatory electrocardiogram monitoring to screen for atrial and ventricular arrhythmias. A strategy of more frequent or prolonged monitoring would lead to earlier arrhythmia recognition and the potential for
\end{abstract}

Hypertrophic cardiomyopathy (HCM) has an approximate prevalence of 1 in 500 in the general population, according to echocardiographic studies. ${ }^{1-3}$ The disease is characterized by left ventricular hypertrophy not attributable to another cardiac, systemic, or metabolic disease. ${ }^{4-6}$ Clinical consequences of HCM include heart failure, atrial fibrillation (AF), stroke, and sudden cardiac death (SCD). Treatment strategies include lifestyle modification, pharmacotherapies, septal reduction therapy, heart transplant, anticoagulation therapy, and implantable cardioverter defibrillators (ICDs) in select patients with HCM. In the modern era, long-term outcomes related to disease-specific mortality are as low as $0.5 \%$ per year. ${ }^{7}$ Low SCD event rates $(<1 \%$ per year in community-based HCM cohorts) in tandem with the heterogenous nature of HCM can make risk stratification challenging in individual patients.

Considerable effort focused on improving the precision of SCD risk prediction in HCM has culminated in the latest

Received for publication October 20, 2021. Accepted January 3, 2022.

Ethics Statement: The research reports in this article has adhered to the relevant ethical guidelines.

Corresponding author: Dr Zachary Laksman, University of British Columbia, Room 211-1033, Davie St, Vancouver, British Columbia, V6E

1M7, Canada. Tel.: +1-604-806-8256; fax: +1-604-806-9274.

E-mail: zlaksman@mail.ubc.ca

See page 412 for disclosure information.

\section{RÉSUMÉ}

La cardiomyopathie hypertrophique (CMH) qui est la cardiomyopathie héréditaire la plus fréquente présente une hétérogénéité clinique importante. La stratification du risque d'arythmies et leur détection sont des composantes essentielles de l'évaluation et de la prise en charge de tous les cas de CMH. Les lignes directrices 2020 de l'American Heart Association et de l'American College of Cardiology en matière de $\mathrm{CMH}$ fournissent les nouvelles recommandations sur la surveillance périodique de l'électrocardiogramme ambulatoire de 2448 heures pour dépister les arythmies auriculaires et ventriculaires.

iteration of the HCM guidelines. ${ }^{4}$ Detection of AF is of critical importance in successful mitigation of the thromboembolic risk with anticoagulation. Likewise, identification of nonsustained ventricular tachycardia (NSVT) remains an important component of SCD risk stratification. The aim of this article is to review current as well as investigative or potential future strategies in arrhythmia detection in patients with HCM and how that may inform treatment strategies and future guidelines.

\section{Arrhythmia Detection}

The primary objectives of arrhythmia monitoring in $\mathrm{HCM}$ are the detection of $\mathrm{AF}$ and ventricular arrhythmias so that appropriate treatment, including anticoagulation and ICD implantation, may be considered. The 2020 American Heart Association/American College of Cardiology (AHA/ACC) guidelines recommend ambulatory electrocardiogram (AECG) monitoring at initial evaluation and periodically, every 1 to 2 years. ${ }^{4}$ Extended 24-hour ECG monitoring or event recording is recommended for patients with palpitations or lightheadedness until symptom-rhythm correlation is established. More frequent and prolonged periods of monitoring lead to a higher detection rate of AF and NSVT in patients with HCM across all risk groups. ${ }^{8,9}$ However, how this monitoring should be incorporated into the evaluation of patients with HCM is not well established. 
appropriate treatment. However, whether such a strategy in patients with $\mathrm{HCM}$ results in improved outcomes is not yet established. The available evidence, knowledge gaps, and potential merits of such an approach are reviewed. Cardiac implantable electronic devices provide an opportunity for early arrhythmia detection, with the potential to enable early management strategies in order to improve outcomes.

In recent decades, an increasing number of cardiac implantable electronic devices (CIEDs), which encompass permanent pacemakers, ICDs, and implantable loop recorders (ILRs), have been used in patients with cardiovascular disease. CIEDs are capable of recording spontaneous episodes of arrhythmias using programmable detection criteria, enabling an estimate of asymptomatic arrhythmia burden in some patient populations. ${ }^{10}$ For example, studies have established a that the prevalence of subclinical AF (SCAF) is high in individuals with these devices. The Asymptomatic Atrial Fibrillation and Stroke Evaluation in Pacemaker Patients and the Atrial Fibrillation Reduction Atrial Pacing Trial (ASSERT) evaluated 2580 patients aged over 65 years who had hypertension without a history of AF. These patients all had either a pacemaker or a defibrillator. Within the first 3 months following implantation, subclinical atrial tachyarrhythmias were detected in $10.1 \%$ of patients and were associated with an increased risk of ischemic stroke or systemic embolism. ${ }^{11}$ In the non-HCM population, an SCAF duration of $>6$ minutes increases the risk of stroke 2.5-fold, ${ }^{11}$ which highlights the importance of prolonged monitoring. However, large variability is present in AF detection algorithms, and sensitivity and specificity across different CIEDs, which may impact their utility in AF detection. Controversy also surrounds the question of whether the components of CIED-detected AF (ie, duration, burden, and $\mathrm{CHA}_{2} \mathrm{DS}_{2}$-VASc [Congestive Heart Failure, Hypertension, Age $\geq 75$ Years, Diabetes Mellitus, Stroke, Vascular Disease, Age 65 to 74 Years, Sex Category] score) are effective predictors of stroke. ${ }^{12}$ Stroke risk is lower in patients with device-detected $\mathrm{AF}$, compared to those with clinical AF with identical stroke risk scores. $^{11,13,14}$

\section{Cardiac monitoring for SCD risk stratification}

Risk stratification for SCD in HCM remains a challenge for clinicians. In both AHA/ACC and European Society of Cardiology (ESC) guidelines, secondary prevention with ICDs is recommended in patients with previous cardiac arrest or hemodynamically significant ventricular tachycardia/ventricular fibrillation (VT/VF) and reasonable life expectancy. ${ }^{4,5}$ Appropriate ICD interventions are 10\%-17\% per year in this population, a level significantly higher compared with that for patients implanted for primary prevention, per retrospective registry data. ${ }^{15-19}$ The low event rates of SCD in HCM present a challenge for primary prevention ICDs. Primary prevention using the European guidelines is based on the calculation of SCD risk within the next 5 years. ${ }^{5,20}$ Routine assessment of risk factors should be performed using
Une stratégie de surveillance plus fréquente et prolongée permettrait de dépister plus précocement l'arythmie et pourrait mener au traitement approprié. Toutefois, il n'a pas encore été établi qu'une telle stratégie chez les patients atteints de $\mathrm{CMH}$ entraînait de meilleurs résultats cliniques. Les données probantes actuelles, les lacunes en matière de connaissances et les mérites potentiels d'une telle approche sont passés en revue. Les dispositifs cardiaques électroniques implantables offrent la possibilité de détecter précocement l'arythmie et le potentiel de favoriser des stratégies de prise en charge précoce pour améliorer les résultats cliniques.

echocardiogram and 48-hour AECG. Variables in the risk calculator include patient age, family history of SCD, personal history of unexplained syncope, left ventricular (LV) outflow gradient, maximum LV wall thickness, left atrial diameter, and NSVT (Fig. 1). ${ }^{21}$ In general, for patients with a 5-year SCD risk less than $4 \%$, ICD implantation is not recommended. Consideration of ICDs should be given to patients with a 5-year SCD risk $\geq 4 \%$.

AHA/ACC guidelines recommend a similar evaluation but add a recommendation for cardiac magnetic resonance imaging to assess for additional risk factors in patients at low or moderate risk. According to the 2020 AHA/ACC guidelines, consideration should be given to patients with HCM with the following risk factors: family history of SCD, maximum LV wall thickness $\geq 30 \mathrm{~mm}$, personal history of unexplained syncope, ejection fraction $\leq 50 \%$, and presence of an apical aneurysm. NSVT and extensive late gadolinium enhancement (LGE) $\geq 15 \%$ of LV mass are also established risk factors used in SCD risk stratification. The details of LGE as a risk factor have been extensively reviewed in the literature. ${ }^{18,22-24}$ NSVT used in risk stratification is based on episodes identified on 24to-48-hour AECG. Neither European nor American risk calculators provide firm recommendations on the detection of NSVT on prolonged monitoring. The use of implantable devices to detect NSVT and AF results in an abundance of data, which currently have little import for change in clinical management. AHA/ACC guidelines suggest that repeated, longer, or faster runs of NSVT should prompt clinicians to consider primary prevention ICDs.

Continuous rhythm monitoring over several years is made possible using implantable devices. These include ILRs, which can help clinicians identify concerning features of ventricular arrhythmias through single-lead electrograms. Patients with HCM and suspicion for arrhythmias but without previously unidentified NSVT or AF may benefit from prolonged monitoring using ILRs. Prior studies in patients with other cardiomyopathies have demonstrated the utility of ILRs in the detection of clinically relevant arrhythmias. ${ }^{25}$ However, data supporting changes in clinical management based on arrhythmias detected using this method are sparse. Despite this lack of data, offering extended monitoring to patients with low ESC risk scores seems reasonable if they have unaccounted risk factors, such as moderate-to-extensive ventricular scarring or apical aneurysms. Other patients who also may benefit include those with moderate SCD risk in which identification of NSVT would put them into the high-SCD risk group. Given recent data correlating faster and longer NSVT episodes with appropriate ICD therapy, ${ }^{26-28}$ patients 


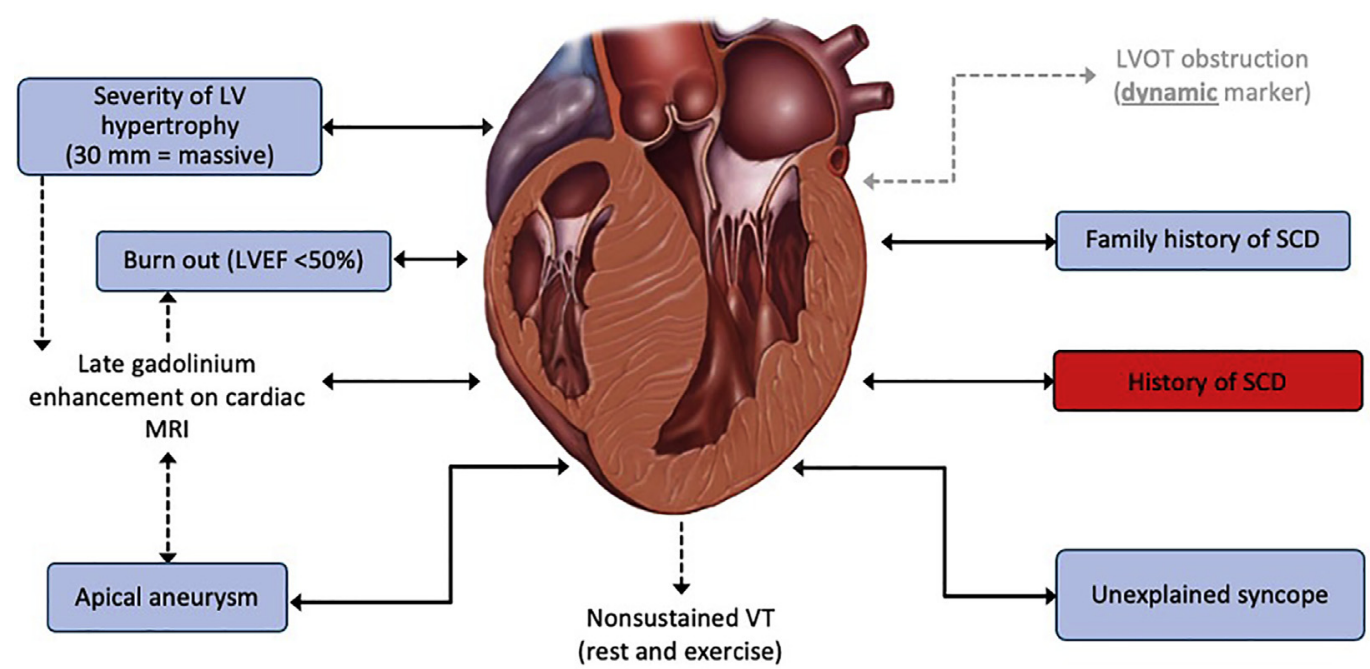

Figure 1. Pathophysiology of hypertrophic cardiomyopathy, and risk factors currently used for risk stratification. LV, left ventricle; LVEF, left ventricular ejection fraction; LVOT, left ventricular outflow tract; MRI, magnetic resonance imaging; SCD, sudden cardiac death; VT, ventricular tachycardia. Reproduced from Geske et al. ${ }^{21}$ with permission from Elsevier.

with previously detected slow or short NSVT runs also may benefit from extended rhythm monitoring. Although current guidelines do not recommend the use of ILRs, a suggested framework for the use of loop recorders for risk stratification is proposed in Figure 2.

No prospective, randomized controlled trials have evaluated the performance of ICDs in HCM, despite their widespread adoption. Mortality rates in HCM were as high as $4 \%-5 \%$ per year prior to the advent of ICD therapy in referral cohorts. ${ }^{29-31}$ The decision to proceed with ICD therapy must be balanced with the risk of inappropriate shocks and other adverse events. A cohort of 217 consecutive patients with HCM followed for over 10 years found that $64 \%$ of patients had ICD discharges occurring $\geq 5$ years after implantation, whereas $54 \%$ of patients experienced inappropriate shocks $<$ 5 years after implantation. ${ }^{32}$ A meta-analysis of 27 observational studies encompassing 2190 patients with HCM across
16 international patient cohorts found appropriate-ICD intervention rates of 3.3\% per year, and low cardiac mortality rates of $0.6 \%$ following ICD therapy. ${ }^{33}$ Inappropriate ICD intervention in this meta-analysis was $4.8 \%$ per year. An updated meta-analysis in 2017 covering 3797 patients found marginally higher appropriate-ICD intervention rates of $4.8 \%$ per year and inappropriate-shock rates of $4.9 \%$ per year. ${ }^{34} \mathrm{~A}$ noteworthy point is that not all ICD discharge events are equivalent to SCD. The decision for primary prevention therapy is further complicated by the relatively young age at which many patients with HCM must first consider implantation. Subcutaneous ICDs are an emerging technology that may have benefits for primary and secondary prevention of SCD in younger patient populations. ${ }^{35} \mathrm{~A}$ single-centre series of subcutaneous ICDs in HCM patients showed that the devices are effective at identifying and terminating induced VF at implantation. ${ }^{36}$

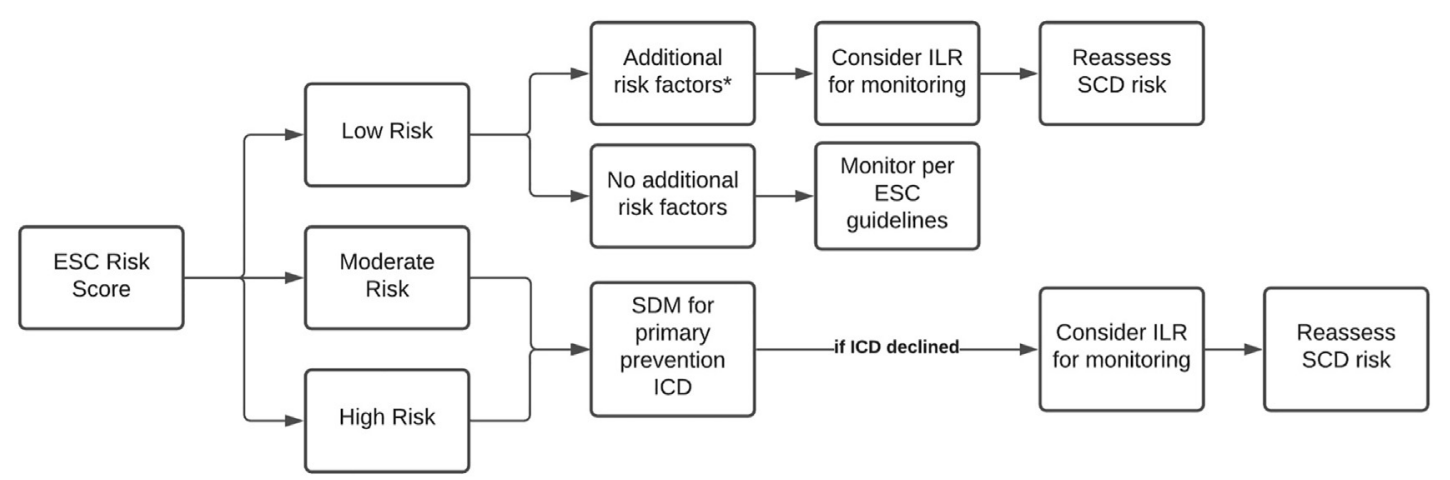

Figure 2. Suggested framework for use of implantable loop recorders (ILRs) for risk stratification of sudden cardiac death (SCD). *Additional risk factors include, but are not limited to, late gadolinium enhancement, apical aneurysms, previous nonsustained ventricular tachycardia episodes with no high-risk features, and genetic variants of concern. ESC, European Society of Cardiology; ICD, implantable cardioverter defibrillator; SDM, shared decision-making. 


\section{AF in HCM}

AF is a common sequela in HCM and is associated with impaired quality of life and increased risk of stroke. ${ }^{37,38}$ Traditional stroke-risk scoring systems for AF (eg, $\mathrm{CHA}_{2} \mathrm{DS}_{2}$-VASc) do not correlate well with clinical outcomes in patients with comorbid HCM and should not be used to assess thromboembolism risk. ${ }^{39,40}$ The HCM riskcerebrovascular accident (CVA) model identified advanced age, New York Heart Association heart failure class III and IV, and left atrial size as risk factors for thromboembolic events. ${ }^{41}$ Large left atrial volume and diameter are sensitive and specific markers for the occurrence of AF in patients with HCM. ${ }^{42,43}$ Recent studies also indicate genetic predisposition, female sex, and the presence of comorbid conditions to be independently related to the occurrence of new-onset AF in HCM. ${ }^{44,45}$ Genetic variations in the $M Y H 7$ and $A C E$ genes have been associated with the development of AF in HCM cohorts. ${ }^{46-48}$ The 2020 AHA/ACC guidelines suggest that patients with HCM who have HCM Risk-CVA model risk factors for AF and are eligible for anticoagulation therapy, should undergo extended AECG monitoring to screen for AF as part of initial evaluation and periodic follow up every 1 to 2 years. ${ }^{4}$ Extended AECG monitoring can be considered for adult patients with HCM who do not have risk factors for AF but are eligible for anticoagulation therapy, in order to screen for asymptomatic paroxysmal $\mathrm{AF}$ at initial evaluation and periodic follow-up every 1 to 2 years. ${ }^{4}$

\section{Traditional device monitoring for AF in HCM}

The prevalence of AF in HCM is 4-to-6-fold higher than it is in the similarly aged general population. Based on 24-48hour AECG monitoring, the overall incidence of AF is $3.1 \%$ per 100 patients per year, with a lifetime prevalence of $22.5 \%$. ${ }^{49}$ A case-control study of 104 patients with HCM showed the presence of AF in approximately $5 \%$ of patients at the time of HCM diagnosis. ${ }^{50}$ An additional $10 \%$ of patients developed the arrhythmia during the 5-year follow-up period. Another study found that AF developed in $22 \%$ of patients with HCM ( $\mathrm{n}=480)$ over 9 years of follow-up. ${ }^{51}$ In these studies, paroxysmal AF was the most common subtype identified, with $42 \%$ of patients with HCM eventually developing chronic persistent or permanent AF.

\section{Implantable device monitoring for AF in HCM}

In a small, high-risk population of 44 patients with HCM with ICDs or pacemakers, the prevalence of AF increased from $32 \%$ to $68 \%$, with 16 patients developing de novo AF during extended monitoring. ${ }^{52}$ Another retrospective study in a separate cohort of 132 patients with HCM with ICDs or pacemakers found an annualized incidence of device-detected AF of $7.0 \%{ }^{53}$ Device-detected AF, defined as symptom-free episodes of rapid atrial rates lasting more than 30 seconds, led to changes in clinical management for most patients in these 2 studies. These contemporary studies also suggest that SCAF is more prevalent than previously thought; however, these studies represented highly selected populations, and the true prevalence of AF in the overall HCM population remains unknown. Table 1 shows AF prevalence and incidence across several studies, as detected by AECG, compared with prolonged rhythm-monitoring devices.

Current guidelines recommend lifelong therapy with a direct oral anticoagulant as the first-line therapy in all patients with $\mathrm{HCM}$ and $\mathrm{AF}$, regardless of $\mathrm{CHA}_{2} \mathrm{DS}_{2}$-VASc score. Individuals with HCM and SCAF detected by internal or external cardiac devices and lasting $>24$ hours should receive anticoagulant therapy. ${ }^{4,5}$ Contemporary studies support this approach. ${ }^{8,37,54}$ The role of anticoagulation is less clear in patients with device-detected SCAF with an episode duration of $>5$ minutes but $<24$ hours. In these cases, duration of the AF episodes, total AF burden, underlying risk factors, and bleeding risk should all be taken into consideration. ${ }^{4}$ Prolonged ambulatory monitoring with an ILR or wearable devices could be a consideration for patients with HCM with a high pretest probability for AF and consequent thromboembolic risk. Consumer-level external cardiac monitoring devices, such as smartwatches and pocket ECG monitoring devices, as they become more accessible, may soon play a larger role in AF detection. These devices would allow for earlier detection of $\mathrm{AF}$ and prompt initiation of appropriate treatment strategies to reduce the risk of stroke and heart failure. Of note, a recent study of 302 consecutive Canadian patients found AF to be a significant predictor of ventricular tachyarrhythmias leading to ICD therapy in patients with HCM. ${ }^{55}$

\section{NSVT in HCM}

NSVT is a common arrhythmia used to estimate suddendeath risk and determine suitability for ICD therapy in both the $\mathrm{ESC}^{5}$ and $\mathrm{AHA} / \mathrm{ACC}^{4}$ guidelines. Most studies define NSVT as $\geq 3$ consecutive beats at $\geq 120$ beats per minute (bpm) and lasting $<30$ seconds. However, this definition uses a low threshold for NSVT and does not appear to confer the same degree of risk in an older vs a younger HCM patient. The presence of NSVT is a class IIb recommendation in adults but a class IIa recommendation in children in the 2020 ACC/AHA guidelines. ${ }^{4}$ Longer, faster, and more-frequent episodes of NSVT are felt to confer increased risk also. However, a noteworthy point is that earlier investigations into

Table 1. Ambulatory electrocardiogram (ECG)-detected atrial fibrillation (AF) prevalence, compared with that detected by prolonged rhythmmonitoring devices

\begin{tabular}{|c|c|c|c|c|}
\hline Study & Patients, n & Prolonged monitoring device (n) & Detected AF prevalence, $\%$ & Detected AF incidence, $\%$ per year \\
\hline Guttmann et al. ${ }^{39}$ & 7381 & 24-48-h Holter & 23 & 3 \\
\hline Olivotto et al. ${ }^{51}$ & 480 & Office ECG & 22 & 2 \\
\hline Wilke et al. ${ }^{52}$ & 44 & ICD (38), PM (5), or ILR (1) & 33 & 53 (over 595-d median follow-up) \\
\hline van Velzen et al. ${ }^{53}$ & 132 & ICD $(116)$ or PM $(16)$ & - & 7 \\
\hline Magnusson and Morner ${ }^{8}$ & 30 & ILR & 30 & - \\
\hline
\end{tabular}

ICD, implantable cardiac device; ILR, implantable loop recorder; PM, pacemaker. 
these variables based on 24- and 48-hour AECG monitoring suggested that they have little prognostic value. ${ }^{56,57}$

The updated guidelines acknowledge that more NSVT episodes are detected on prolonged monitoring, but they maintain the prior 2011 guideline recommendations of AECG monitoring every 1 to 2 years. ${ }^{58}$ Several studies have evaluated the frequency of NSVT in intermittent ambulatory monitoring of patients with HCM. In large, unselected, and non-tertiary patient populations, the frequency of the arrhythmia is estimated to be between $20 \%$ and $30 \%$ on $24-$ and 48-hour AECG monitoring, ${ }^{56,57,59}$ compared with less than $4 \%$ in the general population. ${ }^{60}$ In both HCM and general populations, the prevalence of NSVT increases with age. ${ }^{57,60}$ Given the high prevalence of NSVT, in combination with the low rate of SCD in patients with HCM, NSVT has been criticized for having poor positive predictive value. ${ }^{56}$ In a study assessing the impact of arrhythmias in 178 patients with HCM, NSVT detected on 24-hour ambulatory monitoring had negative and positive predictive values for SCD of $95 \%$ and $9 \%$, respectively. ${ }^{56}$

\section{NSVT as a risk factor for SCD}

Several studies evaluating NSVT detected by 24 - and 48hour AECG have determined that it is not a significant independent risk factor for SCD. ${ }^{61-64}$ Both the population of patients included and the statistical power generated may have an impact on the utility of risk markers under study. For example, one study that was unable to find NSVT to be a significant risk marker for SCD included a predominantly low-risk HCM population. ${ }^{64}$ Patients included in this study were either asymptomatic or mildly symptomatic with heart failure symptoms (New York Heart Association class I or II), had no history of syncope, and were not taking any cardioactive medications. In contrast, another study found that NSVT was a significant risk factor when patients had concurrent left ventricular outflow tract obstruction. ${ }^{62}$ In patients with severe outflow tract obstruction $(\geq 90 \mathrm{~mm} \mathrm{Hg})$, NSVT carried a relative risk of 3.84 for SCD on multivariate analysis.

With newer studies and more-refined patient populations, NSVT has been independently associated with an increased risk of SCD in young patients with $\mathrm{HCM},{ }^{57}$ and in those with exercise-induced NSVT. ${ }^{65}$ In 174 patients aged $<30$ years, NSVT was associated with an odds ratio for sudden death of 4.35. Fifteen patients died suddenly in this age group, including 6 of 26 with NSVT. Exercise-induced NSVT is a rare finding, but it has also been identified as an independent marker for increased risk of SCD. ${ }^{65}$ A total of 24 of 1380 patients with HCM experienced exercise-induced NSVT during Bruce or modified-Bruce protocols. Exercise-NSVT alone was associated with a 2.82-fold increased risk of SCD or resuscitated ventricular arrhythmia. Both of these studies that identified NSVT as an independent marker of SCD are limited by low event rates, and both studies were conducted at a single tertiary care centre. A study from 1981 found that SCD or cardiac arrest occurred in 4 of 17 patients with NSVT, compared with 2 of 66 patients without recorded NSVT on 24-hour ambulatory monitoring. ${ }^{66}$ However, this study predated much of our current understanding about risk factors in HCM.

The prevalence of NSVT is likely underestimated due to the brevity of ambulatory monitoring resulting in missed 

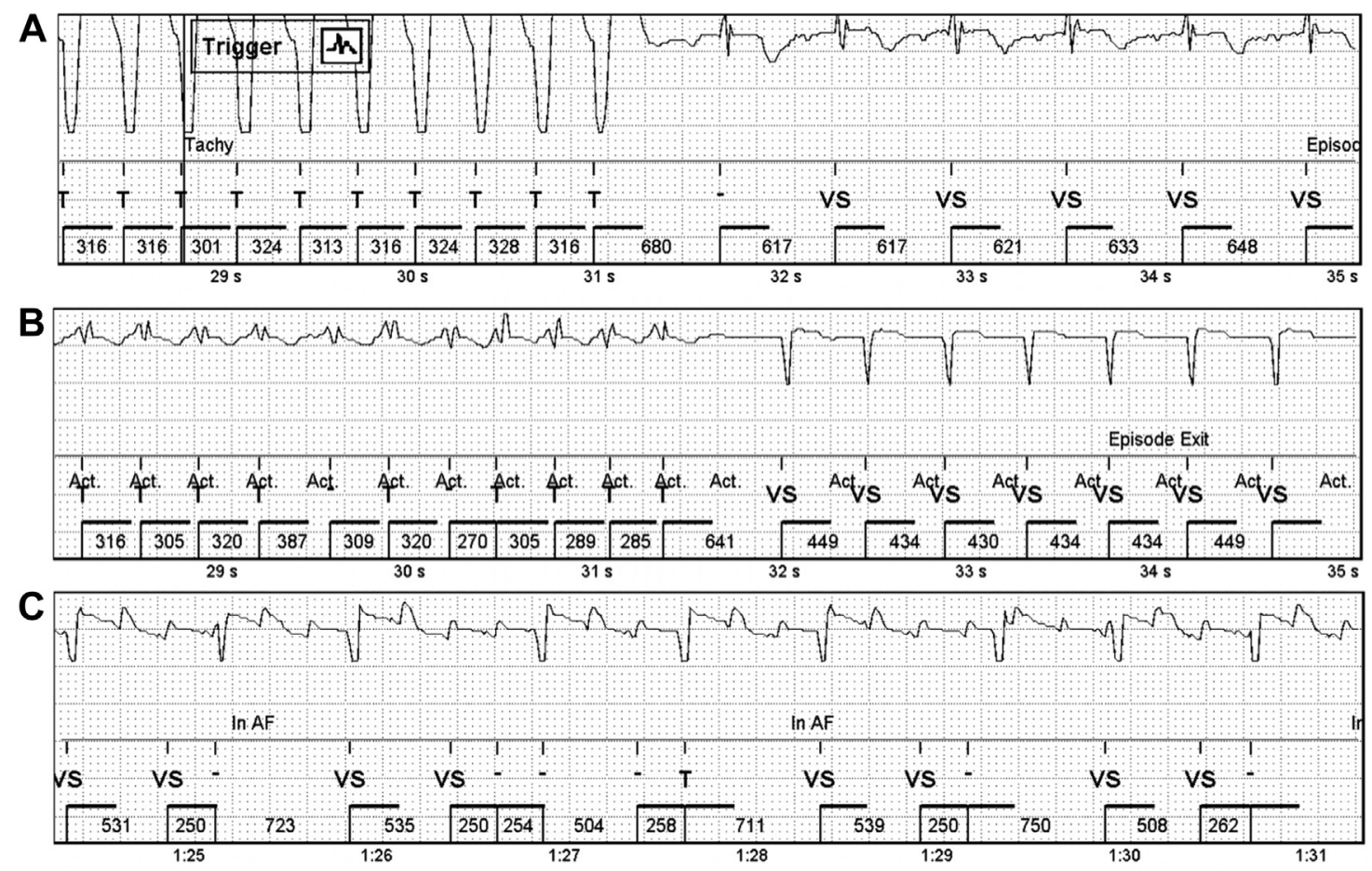

Figure 3. (A, B) nonsustained ventricular tachycardia electrogram episodes. (C) Atrial flutter (AF) electrogram, detected by the Confirm Rx Implantable Cardiac Monitor (Abbott, Chicago) in 3 different hypertrophic cardiomyopathy patients being monitored for risk stratification. Numbers denote milliseconds between beats. T, tachycardia; VS, ventricular sensing; Act, recording activation.

episodes. Table 2 shows NSVT rates detected by 24- to 48 hour Holter monitoring, compared to those detected by prolonged rhythm-monitoring devices, with comparison between detection rates and SCD risk populations. NSVT detection on ILR is lower in several studies, as the rate and interval (number of beats) required for automatic detection are higher, compared with those for Holter monitoring. Contemporary extended monitoring studies evaluating arrhythmias detected in patients with an ICD suggest that the prevalence of NSVT in this high-risk population is upward of $80 \%{ }^{26-28}$ High-risk patients with HCM likely suffer from a higher burden of ventricular arrhythmias, as the risk of sudden death increases along with increased wall thickness. ${ }^{67,68}$ Given the high prevalence of NSVT on prolonged monitoring, its characteristics have been investigated more thoroughly, including rate, length, and frequency. One study of $160 \mathrm{pa}-$ tients using ICD data concluded that NSVT was independently associated with ICD-treated ventricular arrhythmias. ${ }^{27}$ In particular, NSVT rates greater than $200 \mathrm{bpm}$, those with more-than-7-beat runs, and repetitive runs (defined as $>1$ run over $\geq 6$ months of monitoring) were more predictive of ICD-treated ventricular arrhythmias. ICD treatment in this study included anti-tachycardia pacing for VT in 10 patients, and shocks for VT/VF in 14 patients. The average VT zone in the study was $202 \pm 13 \mathrm{bpm}$, and the average VF zone was $233 \pm 13 \mathrm{bpm}$. On ICD interrogation, the mean number of beats was 14 at a mean rate of $222 \mathrm{bpm}$, compared with 11 beats at a mean rate of $152 \mathrm{bpm}$ on AECG monitoring.

Another observational study found evidence that longer duration and faster NSVT episodes are predictive of appropriate ICD intervention for VT/VF, although no cutoff was proposed. ${ }^{26}$ In this study, 41 of 51 total patients had Holter- and/or ICD-recorded NSVT. Of these patients, 31 had NSVT recorded on their ICD across a median of 32 months. Ten patients with previously recorded Holter NSVT did not have any episodes of ICD-recorded NSVT (29\%); by comparison, 7 of 17 patients (41\%) with no prior Holter-NSVT then had at least one episode of ICD-detected NSVT. A large proportion of patients in this study had NSVT episodes detected by Holter monitoring prior to ICD implantation (67\%). All patients enrolled in this study had either one major risk factor for SCD and advanced HCM phenotype, or multiple major risk factors. Authors in this study derived an equation to predict NSVT severity (heart rate $\mathrm{x}$ length in beats/100 $>28$ ), with a hazard ratio of 5.45 for ICD intervention.

Another contemporary study involving 60 patients with prophylactic ICD therapy found that rapid NSVT $(>150$ bpm lasting $4-16$ beats) detected by ICD carried a hazard ratio of $6.2(P=0.01)$ for device-treated ventricular arrhythmias. ${ }^{28}$ Of patients who went on to have ventricular arrhythmias in this study, 7 of 9 also had ICD-detected rapid NSVT. The following aspects are consistent across all these studies that evaluated NSVT detected on prolonged monitoring via ICD: the identification of patients without prior NSVT on AECG; the identification of faster and longer runs of NSVT; and the finding that ICD-detected NSVT is significantly associated with ventricular arrhythmias leading to antitachycardia pacing and/or shock treatment by ICD. As a caveat, many of these earlier studies used antitachycardia pacing and shock treatment by ICD as a surrogate for SCD. The Primary Prevention Parameters Evaluation (PREPARE), Role of Long Detection 
Window Programming in Patients With Left Ventricular Dysfunction, Non-Ischemic Etiology in Primary Prevention Treated With a Biventricular ICD (RELEVANT), and Multicenter Automatic Defibrillator Implantation TrialReduce Inappropriate Therapy (MADIT-RIT) studies suggest that high-rate therapy (ie, faster heart rate detection and longer delays to therapy) reduces the rate of inappropriate therapy, as VT episodes likely will resolve without intervention. $^{69-71}$

\section{Traditional device monitoring for NSVT in HCM}

One retrospective study comparing the diagnostic yield of 14-day Holter monitoring to traditional 24-48-hour monitoring found that $75 \%$ of patients with HCM had NSVT detected during 14-day monitoring. ' Only 17\% of the 77 patients enrolled in this study had NSVT detected in the first 24 hours, and $34 \%$ did in the first 48 hours. Patients who exhibited NSVT episodes in the first 48 hours were more likely to have future episodes of NSVT, with a median number of 5 runs throughout the 14 days. These same patients had longer and faster runs of NSVT. Longer and faster runs were frequently detected beyond the initial 48 hours of monitoring. Only $9 \%$ of patients with runs $>10$ beats were identified in the first 48 hours, compared with $37.7 \%$ of patients in whom these longer runs occurred. Most patients recruited to this study had one or more major risk factors for SCD, and many patients also had moderate or severe LGE on cardiac magnetic resonance imaging. Most patients underwent 14-day Holter testing for the purpose of risk stratification, and thus this study population was created with considerable referral bias. No patient outcome data were assessed in this study; therefore, whether the presence of NSVT on 14-day ambulatory monitoring has any prognostic implications is unclear. However, this monitoring may provide additional risk stratification if faster and longer episodes are detected. A study of 60 patients with primary prevention ICD found that Holter-detection alone was not a significant predictor of ICDtreated ventricular arrhythmia events. ${ }^{28}$

\section{Implantable device monitoring for NSVT in HCM}

ILRs present a viable option for continuous rhythm monitoring in patients with HCM who do not meet criteria for a primary prevention ICD. ILRs are currently recommended for evaluating patients at high risk of developing ventricular arrhythmias, such as those with recurrent unexplained syncope or recurrent palpitations without a diagnosis. ${ }^{2}$ A meta-analysis comprising 4381 patients undergoing ILR insertion for undetermined syncope revealed a diagnosis in $43.9 \%$ of patients. Important to note is that the rate of ventricular arrhythmias diagnosed in the study was $2.7 \%$, despite the exclusion in most studies of patients suspected of having VT/VF. ${ }^{73}$

Two studies have evaluated the use of ILRs in patients with HCM after alcohol septal ablation (ASA). This procedure can theoretically induce ventricular arrhythmias through induction of myocardial scarring. One study in 44 low-risk patients post-ASA found sustained VT/VF in 3 patients within 30 days of ASA, including 2 cases of procedural VF. ${ }^{74}$ Another study evaluating all arrhythmias (supraventricular tachycardia, AF, NSVT, VT, complete heart block) in 56 patients found that the first occurrence of any arrhythmia was $71 \%$ at 18 months of follow-up, and $43 \%$ at 3 months of ASA. ${ }^{75}$ NSVT and VT were detected in 5 patients (9\%).

A study published in 2020 that evaluated ILRs in a lowrisk HCM population detected NSVT in 7 of 30 patients $(23 \%)$, with new detection in 5 patients with previously undetected NSVT on Holter monitoring. ${ }^{8}$ No episodes of VT/ VF were detected in this study. Figure 3 shows NSVT and atrial flutter electrograms recorded by the same type of ILR used in this study. A similar recent study in the Netherlands included 50 low- or intermediate-risk patients with HCM. ${ }^{76}$ Half of the patients received an ILR. Continuous monitoring detected de novo AF only in the ILR group, and one patient from each of the 2 groups received a primary prevention ICD. This study demonstrates the utility of extended continuous monitoring resulting in actionable events, although studies have yet to demonstrate a change in patient outcomes.

\section{Conclusion}

Arrhythmia risk stratification and detection in HCM are critical components of disease evaluation and management. Patients with HCM are at an increased risk of developing arrhythmias, including AF and NSVT, which are associated with increased morbidity and mortality. Current HCM guidelines recommend monitoring for these arrhythmias on an annual basis, using AECG for those without an ICD. Early detection of arrhythmias in HCM prompts initiation of appropriate medical and device therapies, thereby preventing fatal sequelae of the disease. SCAF is better detected with CIEDs, as demonstrated by studies showing increased incidence of $\mathrm{AF}$ on prolonged monitoring, compared with traditional 24- to 48-hour ambulatory monitoring. A similar pattern appears with NSVT on prolonged monitoring. Emerging studies suggest that faster and longer NSVT episodes are more predictive of SCD. However, data supporting a change in management based on NSVT episodes detected on ILR or other prolonged monitoring methods remain limited. Overall, a general clinical trend and mounting evidence support longer and more-intensive outpatient electrocardiographic monitoring in patients with HCM who do not have implantable devices. However, a need remains to demonstrate that this expensive and resource-intensive strategy can translate into improved patient outcomes.

\section{Funding Sources}

Funding for this work was provided by a Health Professional - Investigator Award by the Michael Smith Foundation for Health Research.

\section{Disclosures}

The authors have no conflicts of interest to disclose.

\section{References}

1. Maron BJ. Clinical course and management of hypertrophic cardiomyopathy. N Engl J Med 2018;379:1977.

2. McKenna WJ, Judge DP. Epidemiology of the inherited cardiomyopathies. Nat Rev Cardiol 2021;18:22-36. 
3. Maron BJ, Gardin JM, Flack JM, et al. Prevalence of hypertrophic cardiomyopathy in a general population of young adults. Echocardiographic analysis of 4111 subjects in the CARDIA Study. Coronary Artery Risk Development in (Young) Adults. Circulation 1995;92:785-9.

4. Ommen SR, Mital S, Burke MA, et al. 2020 AHA/ACC guideline for the diagnosis and treatment of patients with hypertrophic cardiomyopathy: a report of the American College of Cardiology/American Heart Association Joint Committee on Clinical Practice Guidelines. Circulation 2020;142:e553-7.

5. Authors/Task Force members, Elliott PM, Anastasakis A, et al. 2014 ESC guidelines on diagnosis and management of hypertrophic cardiomyopathy: the Task Force for the Diagnosis and Management of Hypertrophic Cardiomyopathy of the European Society of Cardiology (ESC). Eur Heart J 2014;35:2733-79.

6. Elliott P, Andersson B, Arbustini E, et al. Classification of the cardiomyopathies: a position statement from the European Society of Cardiology Working Group on Myocardial and Pericardial Diseases. Eur Heart J 2008;29:270-6.

7. Maron BJ, Rowin EJ, Casey SA, et al. Hypertrophic cardiomyopathy in adulthood associated with low cardiovascular mortality with contemporary management strategies. J Am Coll Cardiol 2015;65:1915-28.

8. Magnusson P, Morner S. EvaLuation Using Cardiac Insertable Devices And TelephonE in Hypertrophic Cardiomyopathy (ELUCIDATE HCM): a prospective observational study on incidence of arrhythmias. J Cardiovasc Electrophysiol 2021;32:129-35.

9. Weissler-Snir A, Chan RH, Adler A, et al. Usefulness of 14-day Holter for detection of nonsustained ventricular tachycardia in patients with hypertrophic cardiomyopathy. Am J Cardiol 2016;118:1258-63.

10. Noseworthy PA, Kaufman ES, Chen LY, et al. Subclinical and devicedetected atrial fibrillation: pondering the knowledge gap: a scientific statement from the American Heart Association. Circulation 2019;140: e944-63.

11. Healey JS, Connolly SJ, Gold MR, et al. Subclinical atrial fibrillation and the risk of stroke. N Engl J Med 2012;366:120-9.

12. Mahajan R, Perera T, Elliott AD, et al. Subclinical device-detected atrial fibrillation and stroke risk: a systematic review and meta-analysis. Eur Heart J 2018;39:1407-15.

13. Gage BF, Waterman AD, Shannon W, et al. Validation of clinical classification schemes for predicting stroke: results from the National Registry of Atrial Fibrillation. JAMA 2001;285:2864-70.

14. Chen-Scarabelli C, Scarabelli TM, Ellenbogen KA, Halperin JL. Devicedetected atrial fibrillation: what to do with asymptomatic patients? J Am Coll Cardiol 2015;65:281-94.

15. Maron BJ, Maron MS. Contemporary strategies for risk stratification and prevention of sudden death with the implantable defibrillator in hypertrophic cardiomyopathy. Heart Rhythm 2016;13:1155-65.

16. Maron BJ, Spirito P, Shen WK, et al. Implantable cardioverterdefibrillators and prevention of sudden cardiac death in hypertrophic cardiomyopathy. JAMA 2007;298:405-12.

17. Maron BJ, Spirito P, Ackerman MJ, et al. Prevention of sudden cardiac death with implantable cardioverter-defibrillators in children and adolescents with hypertrophic cardiomyopathy. J Am Coll Cardiol 2013;61: 1527-35.

18. Maron MS, Rowin EJ, Wessler BS, et al. Enhanced American College of Cardiology/American Heart Association strategy for prevention of sudden cardiac death in high-risk patients with hypertrophic cardiomyopathy. JAMA Cardiol 2019;4:644-57.
19. Jayatilleke I, Doolan A, Ingles J, et al. Long-term follow-up of implantable cardioverter defibrillator therapy for hypertrophic cardiomyopathy. Am J Cardiol 2004;93:1192-4.

20. O’Mahony C, Jichi F, Pavlou M, et al. A novel clinical risk prediction model for sudden cardiac death in hypertrophic cardiomyopathy (HCM risk-SCD). Eur Heart J 2014;35:2010-20.

21. Geske JB, Ommen SR, Gersh BJ. Hypertrophic cardiomyopathy: clinical update. JACC Heart Fail 2018;6:364-75.

22. O'Mahony C, Elliott P, McKenna W. Sudden cardiac death in hypertrophic cardiomyopathy. Circ Arrhythm Electrophysiol 2013;6:443-51.

23. Steriotis AK, Sharma S. Risk stratification in hypertrophic cardiomyopathy. Eur Cardiol 2015;10:31-6.

24. Christiaans I, van Engelen K, van Langen IM, et al. Risk stratification for sudden cardiac death in hypertrophic cardiomyopathy: systematic review of clinical risk markers. Europace 2010;12:313-21.

25. Weidemann F, Maier SK, Stork S, et al. Usefulness of an implantable loop recorder to detect clinically relevant arrhythmias in patients with advanced Fabry cardiomyopathy. Am J Cardiol 2016;118:264-74.

26. Francia P, Santini D, Musumeci B, et al. Clinical impact of nonsustained ventricular tachycardia recorded by the implantable cardioverterdefibrillator in patients with hypertrophic cardiomyopathy. J Cardiovasc Electrophysiol 2014;25:1180-7.

27. Wang W, Lian Z, Rowin EJ, et al. Prognostic implications of nonsustained ventricular tachycardia in high-risk patients with hypertrophic cardiomyopathy. Circ Arrhythm Electrophysiol 2017;10:e004604.

28. Viswanathan K, Suszko AM, Das M, et al. Rapid device-detected nonsustained ventricular tachycardia in the risk stratification of hypertrophic cardiomyopathy. Pacing Clin Electrophysiol 2016;39:642-51.

29. Cannan CR, Reeder GS, Bailey KR, Melton LJ 3rd, Gersh BJ. Natural history of hypertrophic cardiomyopathy. A population-based study, 1976 through 1990. Circulation 1995;92:2488-95.

30. McKenna WJ, Deanfield JE. Hypertrophic cardiomyopathy: an important cause of sudden death. Arch Dis Child 1984;59:971-5.

31. McKenna W, Deanfield J, Faruqui A, et al. Prognosis in hypertrophic cardiomyopathy: role of age and clinical, electrocardiographic and hemodynamic features. Am J Cardiol 1981;47:532-8.

32. Rowin EJ, Burrows A, Madias C, et al. Long-term outcome in high-risk patients with hypertrophic cardiomyopathy after primary prevention defibrillator implants. Circ Arrhythm Electrophysiol 2020;13:e008123.

33. Schinkel AF, Vriesendorp PA, Sijbrands EJ, et al. Outcome and complications after implantable cardioverter defibrillator therapy in hypertrophic cardiomyopathy: systematic review and meta-analysis. Circ Heart Fail 2012;5:552-9.

34. Wang N, Xie A, Tjahjono R, et al. Implantable cardioverter defibrillator therapy in hypertrophic cardiomyopathy: an updated systematic review and meta-analysis of outcomes and complications. Ann Cardiothorac Surg 2017;6:298-306.

35. Weiss R, Knight BP, Gold MR, et al. Safety and efficacy of a totally subcutaneous implantable-cardioverter defibrillator. Circulation 2013;128:944-53.

36. Weinstock J, Bader YH, Maron MS, Rowin EJ, Link MS. Subcutaneous implantable cardioverter defibrillator in patients with hypertrophic cardiomyopathy: an initial experience. J Am Heart Assoc 2016;5:e002488.

37. Rowin EJ, Hausvater A, Link MS, et al. Clinical profile and consequences of atrial fibrillation in hypertrophic cardiomyopathy. Circulation 2017;136:2420-36. 
38. Siontis KC, Geske JB, Ong K, et al. Atrial fibrillation in hypertrophic cardiomyopathy: prevalence, clinical correlations, and mortality in a large high-risk population. J Am Heart Assoc 2014;3:e001002.

39. Guttmann OP, Pavlou M, O'Mahony C, et al. Prediction of thromboembolic risk in patients with hypertrophic cardiomyopathy (HCM Risk-CVA). Eur J Heart Fail 2015;17:837-45.

40. Jung H, Yang PS, Sung JH, et al. Hypertrophic cardiomyopathy in patients with atrial fibrillation: prevalence and associated stroke risks in a nationwide cohort study. Thromb Haemost 2019;119:285-93.

41. Malasana G, Day JD, Bunch TJ. Atrial fibrillation in hypertrophic obstructive cardiomyopathy - antiarrhythmics, ablation and more. J Atr Fibrillation 2009;2:210.

42. Debonnaire P, Joyce E, Hiemstra Y, et al. Left atrial size and function in hypertrophic cardiomyopathy patients and risk of new-onset atrial fibrillation. Circ Arrhythm Electrophysiol 2017;10:e004052.

43. Tani $T$, Tanabe $K$, Ono $M$, et al. Left atrial volume and the risk of paroxysmal atrial fibrillation in patients with hypertrophic cardiomyopathy. J Am Soc Echocardiogr 2004;17:644-8.

44. Guttmann OP, Pavlou M, O'Mahony C, et al. Predictors of atrial fibrillation in hypertrophic cardiomyopathy. Heart 2017;103:672-8.

45. Maron BJ, Maron MS, Semsarian C. Genetics of hypertrophic cardiomyopathy after 20 years: clinical perspectives. J Am Coll Cardiol 2012;60:705-15.

46. Lee SP, Ashley EA, Homburger J, et al. Incident atrial fibrillation is associated with MYH7 sarcomeric gene variation in hypertrophic cardiomyopathy. Circ Heart Fail 2018;11:e005191.

47. Gruver EJ, Fatkin D, Dodds GA, et al. Familial hypertrophic cardiomyopathy and atrial fibrillation caused by Arg663His beta-cardiac myosin heavy chain mutation. Am J Cardiol 1999;83. 13H-8H.

48. Ogimoto A, Hamada M, Nakura J, Miki T, Hiwada K. Relation between angiotensin-converting enzyme II genotype and atrial fibrillation in Japanese patients with hypertrophic cardiomyopathy. J Hum Genet 2002;47:184-9.

49. Guttmann OP, Rahman MS, O’Mahony C, Anastasakis A, Elliott PM. Atrial fibrillation and thromboembolism in patients with hypertrophic cardiomyopathy: systematic review. Heart 2014;100:465-72.

50. Robinson K, Frenneaux MP, Stockins B, et al. Atrial fibrillation in hypertrophic cardiomyopathy: a longitudinal study. J Am Coll Cardiol 1990;15:1279-85.

51. Olivotto I, Cecchi F, Casey SA, et al. Impact of atrial fibrillation on the clinical course of hypertrophic cardiomyopathy. Circulation 2001;104: 2517-24.

52. Wilke I, Witzel K, Munch J, et al. High incidence of de novo and subclinical atrial fibrillation in patients with hypertrophic cardiomyopathy and cardiac rhythm management device. J Cardiovasc Electrophysiol 2016;27:779-84.

53. van Velzen HG, Theuns DA, Yap SC, Michels M, Schinkel AF. Incidence of device-detected atrial fibrillation and long-term outcomes in patients with hypertrophic cardiomyopathy. Am J Cardiol 2017;119: $100-5$.

54. Choi YJ, Choi EK, Han KD, et al. Temporal trends of the prevalence and incidence of atrial fibrillation and stroke among Asian patients with hypertrophic cardiomyopathy: a nationwide population-based study. Int J Cardiol 2018;273:130-5.

55. Weissler-Snir A, Dorian P, Rakowski H, Care M, Spears D. Primary prevention implantable cardioverter-defibrillators in hypertrophic cardiomyopathy - Are there predictors of appropriate therapy? Heart Rhythm 2021;18:63-70.

56. Adabag AS, Casey SA, Kuskowski MA, Zenovich AG, Maron BJ. Spectrum and prognostic significance of arrhythmias on ambulatory Holter electrocardiogram in hypertrophic cardiomyopathy. J Am Coll Cardiol 2005;45:697-704.

57. Monserrat L, Elliott PM, Gimeno JR, et al. Non-sustained ventricular tachycardia in hypertrophic cardiomyopathy: an independent marker of sudden death risk in young patients. J Am Coll Cardiol 2003;42:873-9.

58. Gersh BJ, Maron BJ, Bonow RO, et al. 2011 ACCF/AHA guideline for the diagnosis and treatment of hypertrophic cardiomyopathy: a report of the American College of Cardiology Foundation/American Heart Association Task Force on Practice Guidelines. Developed in collaboration with the American Association for Thoracic Surgery, American Society of Echocardiography, American Society of Nuclear Cardiology, Heart Failure Society of America, Heart Rhythm Society, Society for Cardiovascular Angiography and Interventions, and Society of Thoracic Surgeons. J Am Coll Cardiol 2011;58:e212-60.

59. Elliott PM, Gimeno JR, Thaman R, et al. Historical trends in reported survival rates in patients with hypertrophic cardiomyopathy. Heart 2006;92:785-91.

60. Marine JE, Shetty V, Chow GV, et al. Prevalence and prognostic significance of exercise-induced nonsustained ventricular tachycardia in asymptomatic volunteers: BLSA (Baltimore Longitudinal Study of Aging). J Am Coll Cardiol 2013;62:595-600.

61. Elliott PM, Poloniecki J, Dickie S, et al. Sudden death in hypertrophic cardiomyopathy: identification of high risk patients. J Am Coll Cardiol 2000;36:2212-8.

62. Elliott PM, Gimeno JR, Tome MT, et al. Left ventricular outflow tract obstruction and sudden death risk in patients with hypertrophic cardiomyopathy. Eur Heart J 2006;27:1933-41.

63. Kofflard MJ, Ten Cate FJ, van der Lee C, van Domburg RT. Hypertrophic cardiomyopathy in a large community-based population: clinical outcome and identification of risk factors for sudden cardiac death and clinical deterioration. J Am Coll Cardiol 2003;41:987-93.

64. Spirito P, Rapezzi C, Autore C, et al. Prognosis of asymptomatic patients with hypertrophic cardiomyopathy and nonsustained ventricular tachycardia. Circulation 1994;90:2743-7.

65. Gimeno JR, Tome-Esteban M, Lofiego C, et al. Exercise-induced ventricular arrhythmias and risk of sudden cardiac death in patients with hypertrophic cardiomyopathy. Eur Heart J 2009;30:2599-605.

66. Maron BJ, Savage DD, Wolfson JK, Epstein SE. Prognostic significance of 24 hour ambulatory electrocardiographic monitoring in patients with hypertrophic cardiomyopathy: a prospective study. Am J Cardiol 1981;48:252-7.

67. Spirito P, Bellone P, Harris KM, et al. Magnitude of left ventricular hypertrophy and risk of sudden death in hypertrophic cardiomyopathy. N Engl J Med 2000;342:1778-85.

68. Elliott PM, Gimeno Blanes JR, Mahon NG, Poloniecki JD, McKenna WJ. Relation between severity of left-ventricular hypertrophy and prognosis in patients with hypertrophic cardiomyopathy. Lancet 2001;357:420-4.

69. Wilkoff BL, Williamson BD, Stern RS, et al. Strategic programming of detection and therapy parameters in implantable cardioverterdefibrillators reduces shocks in primary prevention patients: results from the PREPARE (Primary Prevention Parameters Evaluation) study. J Am Coll Cardiol 2008;52:541-50. 
70. Gasparini M, Menozzi C, Proclemer A, et al. A simplified biventricular defibrillator with fixed long detection intervals reduces implantable cardioverter defibrillator (ICD) interventions and heart failure hospitalizations in patients with non-ischaemic cardiomyopathy implanted for primary prevention: the RELEVANT [Role of long dEtection window programming in patients with LEft VentriculAr dysfunction, Nonischemic eTiology in primary prevention treated with a biventricular ICD] study. Eur Heart J 2009;30:2758-67.

71. Moss AJ, Schuger C, Beck CA, et al. Reduction in inappropriate therapy and mortality through ICD programming. N Engl J Med 2012;367: 2275-83.

72. Kubala M, Aissou L, Traulle S, Gugenheim AL, Hermida JS. Use of implantable loop recorders in patients with Brugada syndrome and suspected risk of ventricular arrhythmia. Europace 2012;14:898-902.
73. Solbiati M, Casazza G, Dipaola F, et al. The diagnostic yield of implantable loop recorders in unexplained syncope: a systematic review and meta-analysis. Int J Cardiol 2017;231:170-6.

74. Balt JC, Wijffels MC, Boersma LV, Wever EF, ten Berg JM. Continuous rhythm monitoring for ventricular arrhythmias after alcohol septal ablation for hypertrophic cardiomyopathy. Heart 2014;100:1865-70.

75. Bleszynski PA, Goldenberg I, Fernandez G, et al. Risk of arrhythmic events after alcohol septal ablation for hypertrophic cardiomyopathy using continuous implantable cardiac monitoring. Heart Rhythm 2021;18: 50-6.

76. Sakhi R, Huurman R, Theuns D, et al. Incremental value of an insertable cardiac monitor in patients with hypertrophic cardiomyopathy with low or intermediate risk for sudden cardiac death. Cardiology 2021;146: 207-12. 